\title{
Detection of Peri-papillary Atrophy and RNFL Defect from Retinal Images
}

\author{
Gopal Datt Joshi ${ }^{1}$, Jayanthi Sivaswamy ${ }^{1}$, Prashanth R. ${ }^{2}$, and \\ S. R. Krishnadas ${ }^{2}$ \\ ${ }^{1}$ IIIT Hyderabad, Hyderabad, India \\ ${ }^{2}$ Aravind Eye Hospital, Madurai, India
}

\begin{abstract}
The task of detecting peri-papillary indicators associated with the glaucoma is challenging due to the high degree of intra-and inter-image variations commonly seen in colour retinal images. The existing approaches based on direct modeling of the region of interest fail to handle such image variations which compromises detection accuracy. In this paper, a novel detection strategy is proposed which exploits the saliency property associated with these indicators. The region of interest is modeled as a region substantially different from the adjacent image regions. This dissimilarity information is derived at the feature level, between the target and its adjacent regions. Based on the proposed strategy, two novel methods are presented for the detection of peri-papillary atrophy and RNFL defect from colour retinal images. Two different datasets have been used to evaluate the performance of developed solutions. The obtained results are encouraging and establish the strength of the proposed strategy in handling high degree of image variations. The preliminary results and comparative evaluation with direct modeling strategy show viability of proposed strategy to be used in the glaucoma assessment task.
\end{abstract}

Keywords: Retinal Image, Glaucoma, Peri-papillary atrophy, RNFL defect

\section{Introduction}

In a retinal image, two kinds of clinical indicators are used for the assessment of glaucoma (see Fig. 1): i) Intra-papillary and ii) Peri-papillary. The former is related to geometric changes inside the optic disk (OD) or papilla, particularly the enlargement of the cup region. Whereas, the latter is related with indicative image changes occurring in the periphery of the OD region which includes peripapillary atrophy (PPA) and retinal nerve fiber layer (RNFL) defect. The cooccurrence of peri-papillary indicators with intra-papillary indicators improves reliability in glaucoma detection and hence of interest in this work. In continuation of our earlier work on intra-papillary indicators[3], this work focuses on the detection of peri-papillary indicators from colour retinal images.

The problem of detecting peri-papillary indicators has received little attention compared to intra-papillary indicators. The few methods reported in literature for the detection of peri-papillary indicators adopt a detection strategy 


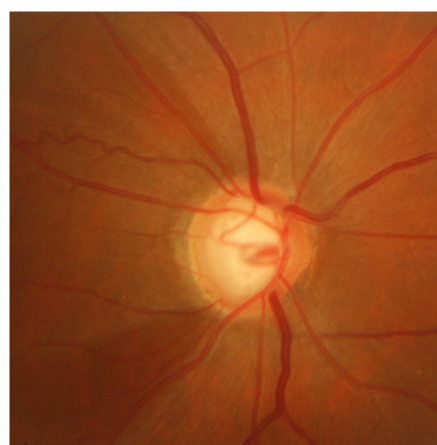

(a)

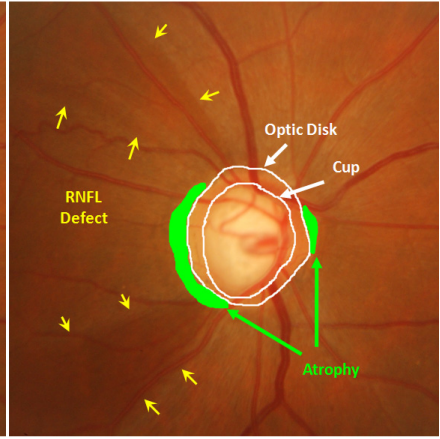

(b)

Fig. 1. Glaucomatous indicators in a colour retinal image. a) sample image and b) marked regions of interest.

primarily based on direct modeling of the region of interest. Here, a local appearance model for the underlying indicator (PPA or RNFL defect) is learned and used to detect the presence of respective indicator in a image region. These appearance models are typically defined either using low-level structural or statistical image features. The decision on the presence of indicator is carried out either using model (template) matching-based approaches or feature-based classification approaches. An overview of individual attempts made for PPA and RNFL defect detection is given below.

To the best of our knowledge, only two attempts have been made towards detecting PPA. First work was reported on Heidelberg retina angiograph images [4][5] in which PPA is modeled as a bright contrasting region. The task of detection (region localisation) is performed using active contours. This method works well on angiographic images where PPA regions appear consistently bright. However, in colour fundus/retinal images (CFI), PPAs appear with varying contrast and texture. Pathological myopia (which is also indicated by the presence of PPAs) detection from such images has been proposed using a variational levelset based active contour to localise candidate PPAs [7][8][13]. The true PPAs are found using spatial and colour based properties which were empirically determined using sample PPA regions.

The detection of RNFL defect was first reported on polarimetric images [14] where the RNFL defect is modeled as a region with straight edges. A dynamic programming-based search is employed to first find such regions and edge strength and shape-based information are used to finally classify the region. This is adapted for colour retinal images in [9] by first enhancing the edges via Gabor filtering before edge-based classification similar to [14]. The classification is performed using linear discriminant analysis and a artificial neural network employing statistical image features including area, edge length, average pixel value of the candidate region and region contrast before and after enhancement. More recently, a texture-based region modeling has been proposed [1][6][10]. This is based on a Gaussian MRF model. The performance of the proposed method was 
also reported using different classifiers. This approach does not consider the role of colour information, possibly due to high inter image variability rendering this information unreliable. In this work, we argue that the direct modeling approach is not an appropriate strategy particularly when underlying region of interest exhibits an inconsistent (signature) profile (either appearance or structural) across retinal images. It yields overlapping representations for both target and normal regions with insufficient discrimination between them.

The local surround should have a role in modeling of peri-papillary indicators in colour retinal images since visual saliency of an individual indicator is strengthened when its local surround is included. It can be observed from Fig. 1 that RNFL defect stands out well relative to its immediate background which equally holds for PPA. However, this separation decreases when local surround is ignored like in earlier modeling strategy. Therefore, we shift the focus of modeling from the lesion to that of the dissimilarity between the lesion and the adjacent regions. Modeling a lesion in the dissimilarity feature space is expected to be minimally affected by inter- and intra-image variations. Based on the above strategy, we present a common approach for detecting PPA and RNFL defect from colour retinal images. In this approach, candidate regions of interest are first extracted along with respective adjacent region. The dissimilarity between a candidate and its adjacent region is then derived in an appropriate feature space. The final classification (detection) is performed in the dissimilarity feature space by combining decisions obtained from multiple classifiers. We evaluate and report the gain in the performance for both lesions over the direct modeling strategy.

The organisation of the paper is as follows. Sections 2 and 3 present our modeling technique for PPA and RNFL defect based on a local context-based detection strategy. The classification scheme based on a combination of classifiers is presented in Section 4. Details of assessment and results are presented in Section 5. We end with a discussion and conclusion.

\section{Modeling of Peri-papillary atrophy}

Since the PPA is a region on the periphery of the OD, the scheme illustrated in Fig. 2(a-1) is used to obtain candidate PPA region and its adjacent region referred as level- $1 \& 2$, respectively. The dissimilarity between level $1 \& 2$ regions of a candidate region is quantified using image features.

Feature Extraction: Prior to extracting image features, vessel regions are smoothed to reduce their effect on the computed dissimilarity value since the distribution of vessels is variable in level $1 \& 2$ image regions. The vessel regions are inpainted by performing selective morphological closing in 8 directions on red, green and blue colour space[3] and the final vessel-free colour retinal image is used for further processing.

Let the tuple $C^{P}, B^{P}$ be the PPA candidate region (level 1) and its associated background image region (level 2). A region is analysed using a set of (vertically

oriented) scan lines and the intensity variations along these scan lines are used to derive a feature vector (see Fig. 2(a-2)). The 1-D intensity profile along each scan 


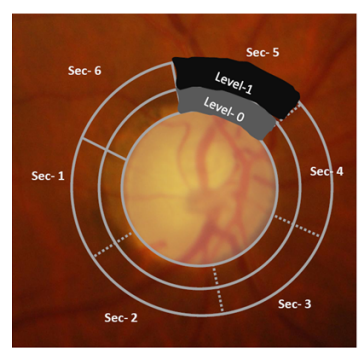

(a-1)

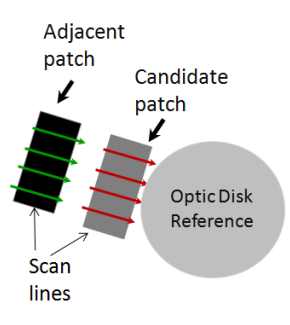

(a-2)

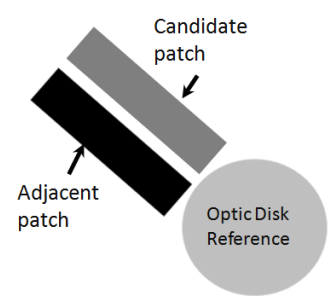

(b)

Fig. 2. Extraction of candidate and adjacent regions for a-1) PPA b) RNFL Defect. a-2) Annular regions divided into wedge shaped regions.

line is used to compute the following statistics: maximum, minimum, average and standard deviation. The final feature vector $f^{P}$ is formed by concatenating the feature vectors for the red, green and blue planes:

$f^{P}=C O N C A T_{r, g, b}^{i}\left\{\max _{i}, \min _{i}, \mu_{i}, \sigma_{i}\right\}$ where $i$ denotes the scan line. Feature vectors are computed for both $C^{P}$ and $B^{P}$. Finally, the dissimilarity between these two regions is found by taking the vector difference between the features as: $x_{P}=f_{B}^{P}-f_{C}^{P}$. The length of $x$ depends on the number of scan lines $i$. In the dissimilarity space, the PPA and normal regions should be well separated.

In order to demonstrate the strength of the proposed modeling, we select the two most discriminant feature dimensions using linear discriminant classifier criteria. This is shown as a scatter plot for both $f_{C}^{P}$ representing the direct modeling space and $x_{P}$ representing the proposed dissimilarity feature space in Fig. 3(a) and (b)). It can be observed from Fig. 3(a) that the PPA (shown in red) and normal (shown in green) clusters overlap quite a bit near the origin and they are more separated as one moves away from the origin. In contrast, the plot in Fig. 3(b) shows that the PPA and normal clusters are better separated. The normal regions are mostly concentrated at the origin whereas PPA regions get values away from the origin representing high deviation of candidate region from its adjacent region.

\section{Modeling of RNFL Defect}

The RNFL defect is an irregular, (roughly) wedge shape region starting from OD. Thus, it is of a greater spatial extent than a PPA region(see Fig. 1). The region is more distinguishable, relative to its background, near the OD region and less so towards the periphery. Since the presence of RNFL defect is of interest rather than a precise segmentation, we model the candidate RNFL region and its associated background as rectangular patches. Similar to PPA detection approach, a feature dissimilarity-based representation is derived using adjacent regions. In addition to vessel smoothing, similar to PPA modeling, we perform anisotropic diffusion [12] based smoothing to obtain a uniform intra-region statistics while preserving points with significant gradient value. Considering OD as 

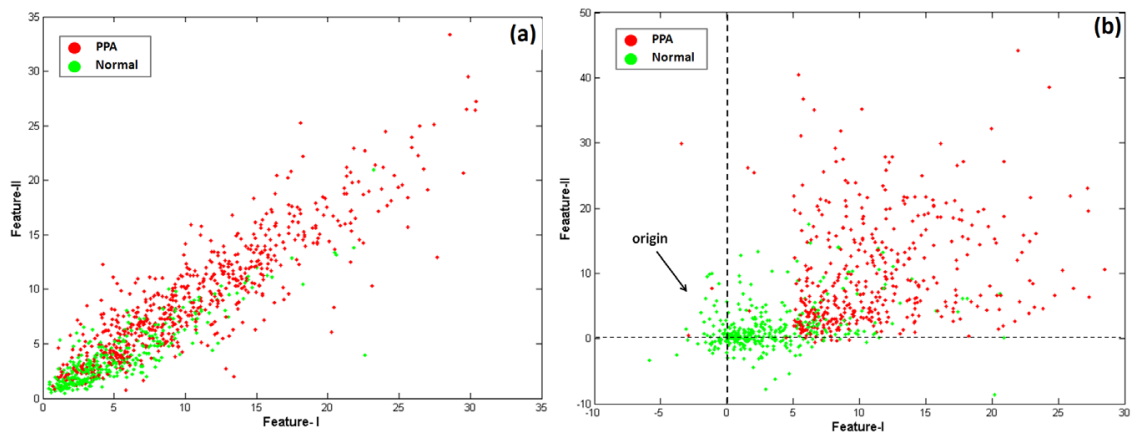

Fig. 3. Different feature spaces obtained for a) Direct Target Modeling b) Proposed Modeling.

a reference, the candidate and adjacent image patches of width $w_{r}$ and length $l_{r}$ are extracted as shown in Fig. 2(b).

Feature Extraction: In order to capture the global visual saliency exhibited by a RNFL defect region, we chose global regional features to derive the representation for a candidate patch. Both colour and texture features are used to capture the global characteristics of an image region. Colour features are formed by two color histograms computed in RGB and Lab colour space. Histograms are quantised into 20 bins and a total 60 colour features are obtained for each colour space. Rotation invariant texture features are obtained in the RGB space using local binary patterns [11] which have been shown to represent local texture well [11]. The final feature vector for a image region $H$ is:

$$
f_{H}^{R}=\left\{\text { hist }_{r g b}, \text { hist }_{L a b}, L B P_{r g b}\right\}
$$

Let $C^{R}$ be a candidate and $B^{R}$ be the associated adjacent image region. The feature vector $x_{R}$ that represents the candidate region is computed as: $x_{R}=$ $f_{B}^{R}-f_{C}^{R}$. The size of $x_{R}$ is fixed. We once again provide a comparison between direct and proposed modeling by computing the two most discriminant feature dimensions for $f_{C}^{R}$ and $x_{R}$. The resulting scatter plots are as shown in Fig. 4 . It can be seen from the scatter plots that proposed strategy did improve the separability in the feature space as in the case of PPA modeling.

\section{Classification}

A classification approach that consists of a set of classifiers and a decision combination function is employed for the detection of both PPA and RNFL defect. Multiple classifiers are used and the final decision on a test image region is derived by combining the decisions obtained from individual classifiers. The following classification schemes were used: k-NN classification, Nearest Mean Scaled Classification, Linear discriminant classification (LDC) in the feature space reduced by principle component analysis, LDC with relevant feature selection (1nearest neighbor error) and LDC with relevant feature selection (leave-one-out error) [2]. The mean, maximum, minimum, median and product combination 

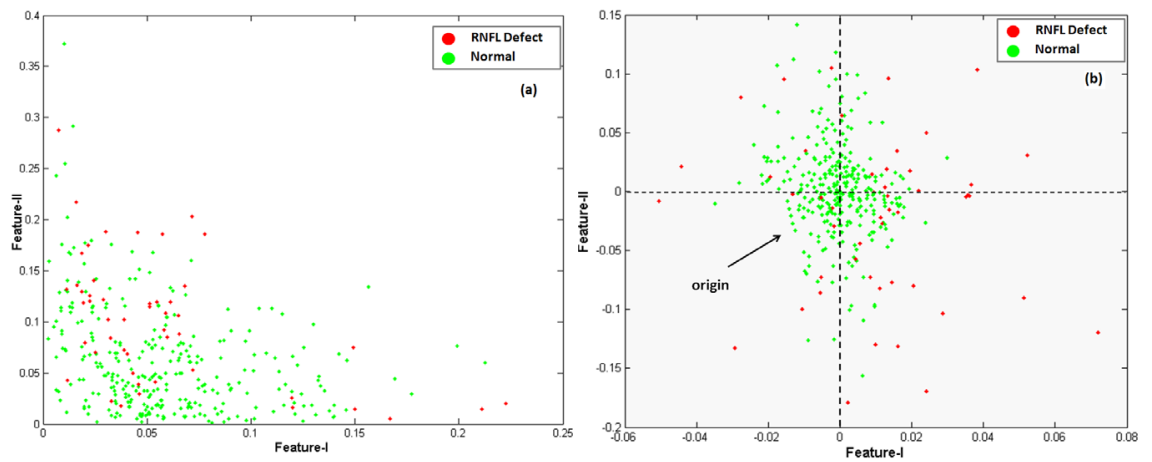

Fig. 4. Discriminant feature spaces for different RNFL defect modeling approaches. a) Direct Target Modeling b) Proposed Modeling.

rules were experimented and the combination with the best performance is reported.

\section{Experiment Results}

The detection performance achieved for each indicator is reported both at region and image-level. The region-level performance reflects the capability of a method in detecting indicators from an image region. The image-level detection sensitivity indirectly suggests utility of a method in detecting glaucoma when combined with intra-papillary analysis results. The strength of the proposal is demonstrated by performing a comparison with the direct modeling strategy.

In the implementation of the direct modeling strategy, only the candidate region is considered for computing features. The experiment specifications were kept the same for a fair comparison. These specifications include: number of training/testing images; type of classifiers and their combination; region dimensions; etc. Two variants of direct modeling strategy were tested. The variation was in the type of features used: a) proposed features - this is referred to as $D M-P, \mathrm{~b})$ MRF-based features - this is referred to as $D M-M$. The MRF-based texture features were used in [6][10][1] for RNFL defect detection where a total of 6 features (MRF parameters) were derived for a image point and used for the classification. Similarly, we compute six features for each point in a candidate region. The average values of these six features computed over the entire candidate region are used to represent a candidate region. The proposed local context-based approach is referred to as $L C-P$.

Dataset: Two datasets of 114 images (for PPA detection) and 25 images (for RNFL defect detection) were collected from a local hospital. These images are OD centric and are in two different sizes $2896 \times 1944$ and $2146 \times 1764$. Ground truth in the form of region markings for PPA was collected. Sector-wise decision on the presence of RNFL defect was collected from a glaucoma expert. PPA dataset was divided into 59 training and 55 testing images and RNFL defect dataset was divided in 15 training and 10 testing images. 


\begin{tabular}{|l|c|c|c|c|c|c|}
\multicolumn{1}{c|}{} & \multicolumn{2}{c|}{ A: PPA Detection } & \multicolumn{3}{c|}{ B: RNFL Defect Detection } \\
\cline { 2 - 7 } \multicolumn{1}{c|}{} & Sensitivity & Specificity & Accuracy & Sensitivity & Specificity & Accuracy \\
\hline DM-M & 0.66 & 0.66 & 0.65 & 0.04 & 0.99 & 0.81 \\
DM-P & 0.67 & 0.65 & 0.66 & 0.23 & 0.92 & 0.79 \\
LC-P & 0.82 & 0.72 & 0.78 & 0.80 & 0.93 & 0.78 \\
\hline
\end{tabular}

Table 1. A) PPA Detection Performance, and B) RNFL Defect Detection Performance.

PPA detection: For an image, 18 image regions, each of width $w_{p}=90$ pixels were automatically extracted around the OD region. This results in 1062 and 990 image regions in the training and testing set, respectively. The test set of PPA contained $637 \mathrm{PPA}$ and 353 normal regions. Classification accuracy along with sensitivity and specificity for PPA detection were computed. The accuracy is defined as: accuracy $=\frac{t p+t n}{\text { total no. of patches }}$. The overall results on PPA test set are presented on Table $1(\mathrm{~A})$. The obtained results indicate that the ability of direct modeling in handling high amount of intra- and inter-image variation is limited. This is consistent across different choice of features for region modeling (see the performance difference of DM-M and DM-P). In contrast, there is a significant gain in the performance is achieved by LC-P with the same set of proposed features. The gain in the accuracy suggests that the chosen strategy is better in separating the normal and PPA regions. At the image-level, LC$\mathrm{P}$ achieves a precision of 0.98 with a recall value of 1 on 55 test images. This indicates that peri-papillary anomaly detection can be reliably used to aid intrapapillary analysis results and comprehensively assess the presence of glaucoma in the retinal images.

RNFL defect detection:The region length $l_{r}$ and width $w_{r}$ were chosen to be 100 and 30 pixels respectively. A total of 14 regions were obtained from an image which results in 225 training and 150 testing image regions. The test set consists of 26 regions with RNFL defect and 124 normal regions. The overall results obtained on this set by different approaches are given in Table 1(B). The DM-M gives very low detection sensitivity and seems to be more selective to the normal cases which is reflected in the obtained accuracy. The DM-P shows gain in the sensitivity which probably indicates the role of colour features which were used in the modeling in addition to the texture features. The higher sensitivity achieved by LC-P again indicates suitability of the proposed strategy in RNFL defect detection. At the image level, LC-P achieves a precision value 0.89 and a recall value 0.89 on the test images. These are lower than PPA attesting to the challenging nature of the problem of RNFL defect detection.

\section{Discussion and Conclusion}

A novel strategy is proposed for the detection of peri-papillary indicators from the retinal images. The strategy is motivated from the saliency exhibited by these indicators in the presence of their local surround. This saliency aspect is represented by using a context-based approach to modeling of an indicator. Two novel solutions for the detection of PPA and RNFL defect were developed under 
this strategy. The preliminary experiment results strongly indicate the superiority of the proposed strategy over the direct modeling strategy adopted in existing solutions. The obtained results also show that the proposed strategy is more robust to intra- and inter-image variations typically seen in retinal images. Future investigations will be towards improvements in the following directions: Optimal selection of dimension for the candidate region and robust feature modeling to better handle inter-image variations.

\section{References}

1. Gazarek, J., Jan, J., Kolar, R., Odstrcilik, J.: Bimodal comparison of retinal nerve fibre layer atrophy evaluation. Proc. Biosignal: Analysis of Biomedical Signals and Images 20, 409-413 (2010)

2. van der Heijden, F., Duin, R.P., de Ridder, D., Tax, D.M.: Classification, parameter estimation and state estimation - an engineering approach using MATLAB. John Wiley \& Sons, ISBN 0470090138 (2004)

3. Joshi, G.D., Sivaswamy, J., Krishnadas, S.R.: Optic disk and cup segmentation from monocular colour retinal images for glaucoma assessment. IEEE Transactions on Medical Imaging (2011)

4. Kolar, R., Jan, J., Kubecka, L.: Computer support for early glaucoma diagnosis based on the fused retinal images. Scripta Medica (BRNO) 79(5-6), 249-260 (2006)

5. Kolar, R., Jan, J., Laemmer, R., Jirik, R.: Semiautomatic detection and evaluation of autofluorescent areas in retinal images. Proc. EMBS pp. 3327-3330 (2007)

6. Kolár̆, R., Vácha, P.: Texture analysis of the retinal nerve fiber layer in fundus images via markov random fields. Proc. IFMBE 25/XI, 247-250 (2009)

7. Lee, B.H., Wong, D.W.K., Tan, N.M., Zhang, Z., Lim, J.H., Li, H., Yin, F., Liu, J., Huang, W., Saw, S.M., Tong, L., Wong, T.Y.: Fusion of pixel and texture features for the detection of pathological myopia. Proc. IEEE Conf. Industrial Electronics and Applications pp. 2039-2042 (2010)

8. Liu, J., Wong, D.W.K., Tan, N.M., Zhang, Z., Lu, S., Lim, J.H., Li, H.: Automatic classification of pathological myopia in retinal fundus images using PAMELA. Proc. SPIE 7624, 76240G: Medical Imaging: Computer-Aided Diagnosis (2010)

9. Muramatsu, C., Hayashi, Y., Sawada, A., Hatanaka, Y., Hara, T., Yamamoto, T., Fujita, H.: Detection of retinal nerve fiber layer defects on retinal fundus images for early diagnosis of glaucoma. Journal of Biomedical Optics 15(1), 016021-1 016021-7 (2010)

10. Odstrcilik, J., Kolar, R., Harabis, V., Gazarek, J., Jan, J.: Retinal nerve fiber layer analysis via markov random fields texture modelling. Proc. EUSIPCO pp. 1650-1654 (2010)

11. Ojala, T., Pietikainen, M., Maenpaa, T.: Multiresolution gray-scale and rotation invariant texture classification with local binary patterns. IEEE Trans. Pattern Analysis and Machine Intelligence 24(7), 971-987 (2002)

12. Perona, P., Malik, J.: Scale-space and edge detection using anisotropic diffusion. IEEE Transactions on Pattern Analysis and Machine Intelligence 12(7), 629-639 (1990)

13. Tan, N., Liu, J., Wong, D., Lim, J., Zhang, Z., Lu, S., Li, H., Saw, S., Tong, L., Wong, T.: Automatic detection of pathological myopia using variational level set. Proc. IEEE EMBS pp. 3609-3612 (2009)

14. Vermeer, K., Vos, F., Lemij, H., Vossepoel, A.: Detecting glaucomatous wedge shaped defects in polarimetric images. Medical Image Analysis 7(4), 503-511 (2003) 